\title{
Cytoreductive Surgery
}

National Cancer Institute

\section{Source}

National Cancer Institute. Cytoreductive Surgery. NCI Thesaurus. Code C132068.

A surgical procedure that refers to the removal of as many malignant cells as possible from an anatomic site affected by cancer. 